Objectives: We hypothesised that if IMB is indeed an RA-feature, then (1) at diagnosis its presence associates with other measures of local inflammation (synovitis, tenosynovitis and osteitis) and (2) it responds to DMARD therapy similarly as these other local inflammatory measures. These hypotheses were tested in a comprehensive MRI-study.

Methods: 157 consecutive early RA patients underwent unilateral contrast-enhanced 1.5T MRI of the forefoot at diagnosis. MRIs were evaluated for presence of IMB and for synovitis, tenosynovitis and osteitis in line with the RA MRI scoring system (summed as RAMRIS-inflammation). MRIs at 4, 12 and 24 months were evaluated for presence and size of IMB-lesions in patients who had IMB at baseline and received early DMARD-therapy. Logistic regression was used for analyses at patient-level; generalised estimating equations were used for bursa-level analyses. Stratification for ACPA was performed.

Results: $69 \%$ of RA patients had $\geq 1 \mathrm{IMB}$. In multivariable analyses on bursa-level, presence of IMB was independently associated with local presence of synovitis and tenosynovitis (OR $1.69(95 \% \mathrm{Cl} 1.12-2.57)$ and 2.83 (1.80-4.44), respectively), but not with osteitis. On patient-level, presence of IMB was most strongly associated with tenosynovitis (OR 2.92 (1.62-5.24)). During treatment with DMARDs, the average size of IMB-lesions decreased (Figure 1). This decrease was associated with decrease in RAMRIS-inflammation scores; most strongly with a decrease in synovitis but not in osteitis. Within ACPA-positive and ACPA-negative RA similar results were obtained.

Conclusion: IMB particularly accompanies inflammation of the synovial lining of joints and tendon-sheaths, both regarding simultaneous occurrence at diagnosis and simultaneous treatment-response. These findings suggest that IMB represents juxta-articular synovial inflammation and indeed is a hallmark of early RA. REFERENCES:

[1] Dakkak YJ et al. Increased frequency of intermetatarsal and submetatarsal bursitis in early rheumatoid arthritis: a large case-controlled MRI study. Arthritis Res Ther 22, 277 (2020).

Disclosure of Interests: None declared.

DOI: 10.1136/annrheumdis-2021-eular.303

\section{POS0022 PHARMACOVIGILANCE PREGNANCY DATA IN A LARGE POPULATION OF PATIENTS WITH CHRONIC INFLAMMATORY DISEASE EXPOSEDTO CERTOLIZUMAB PEGOL: PREGNANCY OUTCOMES AND CONFOUNDERS}

M. Clowse ${ }^{1}$, R. Fischer-Betz ${ }^{2}$, C. Nelson-Piercy ${ }^{3}$, A. Scheuerle ${ }^{4}$, T. Kumke ${ }^{5}$, B. Lauwerys ${ }^{6}$, R. Kasliwal ${ }^{7}$, F. Förger ${ }^{8} .{ }^{1}$ Duke University Medical Center, Division of Rheumatology, Durham, United States of America; ${ }^{2}$ Heinrich Heine University Düsseldorf, Department for Rheumatology, Düsseldorf, Germany; ${ }^{3}$ Guy's and St Thomas' NHS Foundation Trust, Department of Women and Children's Health, London, United Kingdom; ${ }^{4}$ UT Southwestern Medical Center, Department of Pediatrics, Division of Genetics and Metabolism, Dallas, United States of America; ${ }^{5}$ CB Pharma, N/A, Monheim am Rhein, Germany; ${ }^{6} U C B$ Pharma, N/A, Brussels, Belgium; ${ }^{7}$ UCB Pharma, N/A, Slough, United Kingdom; ${ }^{8}$ Inselspital, University of Bern, University Clinic for Rheumatology, Immunology and Allergology, Bern, Switzerland

Background: Chronic inflammatory diseases (CID) in women of reproductive age are increasingly being treated with tumour necrosis factor inhibitors (TNFi), in line with recent guidelines. ${ }^{1}$ However, data on TNFi-exposed pregnancy outcomes are still limited. Certolizumab pegol (CZP), a PEGylated, Fc-free TNFi, has no/minimal placental transfer from mother to infant during the third trimester. ${ }^{2}$

Objectives: To assess pregnancy outcomes from the UCB Pharmacovigilance safety database from over 1,300 prospectively reported pregnancies with maternal CZP exposure.

Methods: Details of CZP-exposed pregnancies from the UCB Pharmacovigilance safety database were reviewed up to November 1,2020. Analysis was limited to prospectively reported cases with known pregnancy outcomes to avoid potential reporting bias. Confounders (specific CID, non-biologic medications and maternal infection) were evaluated using a multivariate stepwise regression model; results from the confounders analysis are reported as odds ratios (OR) with $95 \%$ confidence intervals $(\mathrm{Cl})$. Patients with missing information about presence or absence of confounders were excluded from the model.

Results: 1,392 prospective pregnancies (1,425 fetuses) with maternal CZP exposure and known outcomes were reported (Figure 1). Mean (SD) maternal age was $31.9(5.1)$ years. Of these, $1,021 / 1,392(73.3 \%)$ pregnancies had at least first-trimester CZP exposure and 547/1,392 (39.3\%) were exposed during all trimesters. Overall, there were 1,259/1,425 (88.4\%) live births, $150 / 1,425(10.5 \%)$ abortions (miscarriages and terminations), 11/1,425 (0.8\%) stillbirths, and 5/1,425 (0.4\%) ectopic pregnancies. Congenital malformations were reported in 35/1,425 fetuses $(2.5 \%)$ and in $30 / 1,259$ live-born infants $(2.4 \%) ; 26(2.1 \%)$ congenital malformations were major according to the Metropolitan Atlanta Congenital Defects Program criteria. There was no pattern of specific congenital malformations. Preterm births occurred in 124/1,259 (9.8\%) live births, and 101/1,259 (8.0\%) of infants had low birth weight $(<2.5 \mathrm{~kg})$. In the confounders analysis, reported corticosteroid use was independently associated with increased odds of preterm birth (OR [95\% Cl]: 2.1 [1.3-3.4]; $\mathrm{p}<0.005)$ and low birth weight (OR [95\% Cl]: $1.7[1.0-2.9]$; $p<0.05)$, but decreased odds of abortion (OR [95\% Cl]: 0.5 [0.3-0.9]; $p<0.05)$. Reported NSAID use was associated with increased odds of abortion (OR [95\% Cl]: 2.2 [1.2-4.0]; $\mathrm{p}<0.05$ ), as was methotrexate/leflunomide use (OR [95\% Cl]: 3.2 [1.7-6.2]; $p<0.0005)$. Materna infections were associated with increased odds of preterm birth (OR [95\% Cl]: 1.9 $(1.1-3.5 ; p<0.05)$. Finally, there was an association between a diagnosis of Crohn's disease and odds of abortion (OR [95\% Cl]: 2.5 [1.5-4.1]; $p=0.0005$ ), and between rheumatoid arthritis and low birth weight (OR [95\% Cl]: 1.9 [1.1-3.3]; $p<0.05$ ).

Conclusion: This prospective analysis, including more than 1,000 pregnancies with CZP exposure in at least the first trimester, represents one of the largest cohorts of pregnancies with known outcomes in patients with CID. Our data confirm the impact of specific CID, concomitant drugs or comorbidities on pregnancy outcomes. In particular, additional use of corticosteroids was highlighted as a risk factor for preterm birth and low birth weight in our cohort of CZP-treated patients. No increase in adverse pregnancy outcomes or specific congenital malformations was observed in CZP-exposed pregnancies, compared to the general population, ${ }^{3,4}$ which offers further reassurance for women of childbearing age considering CZP treatment. REFERENCES:

[1] Sammaritano LR. Arthritis Rheum 2020;72:529-56;

[2] Mariette X. Ann Rheum Dis 2018;77:228-33;

[3] Ventura SJ. Natl Vital Stat Rep 2012;60:1-21;

[4] Lee H. BMC Pregnancy Childbirth 2020;20:33.

Figure. Reports of pregnancies exposed to CZP identified in the UCB Pharmacovigilance safety database

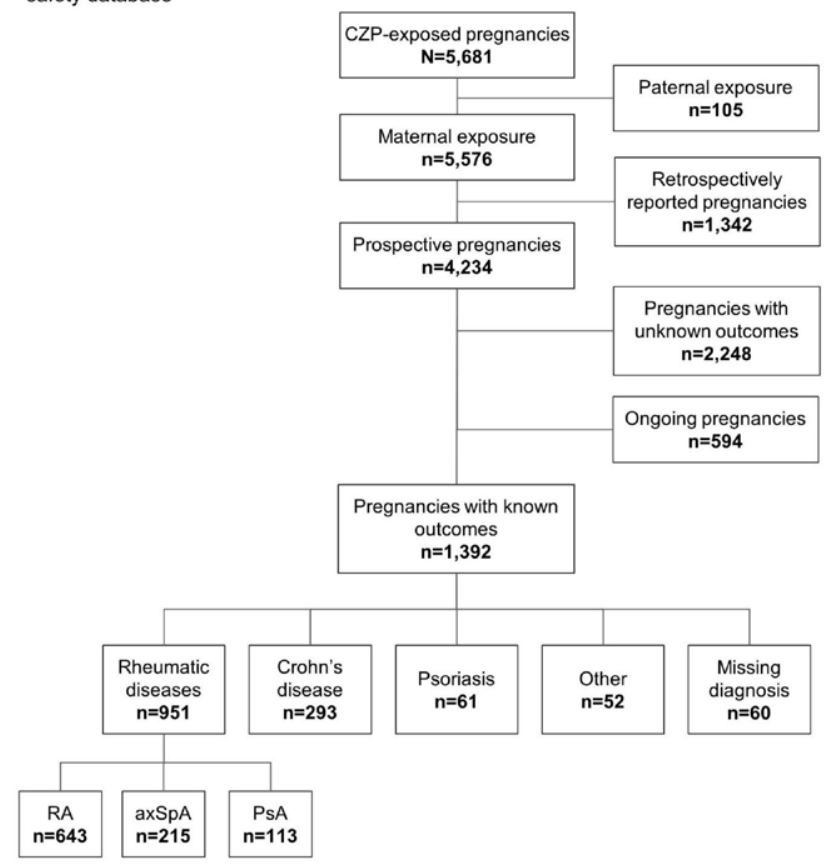

Some patients may have had multiple indications for CZP treatment. axSpA: axial spondyloarthritis; CZP: certolizumab pegol; PsA: psoriatic arthritis; RA: rheumatoid arthritis.

Acknowledgements: This study was funded by UCB Pharma. Editorial services were provided by Costello Medical.

Disclosure of Interests: Megan Clowse Consultant of: UCB Pharma, Grant/ research support from: Janssen, Pfizer, Rebecca Fischer-Betz Consultant of: AbbVie, BMS, Celgene, Chugai, Eli Lilly, Janssen, Novartis, Pfizer, Sanofi, UCB Pharma, Catherine Nelson-Piercy Consultant of: Alliance Pharma, UCB Pharma Angela Scheuerle Consultant of: Antiretroviral Pregnancy Registry, Harmony Biosciences, IQVIA, ICON, NovoNordisk, PPD, Roche, Sanofi-Genzyme, Syneos, UCB Pharma, ViiV, Thomas Kumke Shareholder of: UCB Pharma, Employee of: UCB Pharma, Bernard Lauwerys Employee of: UCB Pharma, Rachna Kasliwa Employee of: UCB Pharma, Frauke Förger Speakers bureau: Mepha, Roche, UCB Pharma, Grant/research support from: UCB Pharma.

DOI: 10.1136/annrheumdis-2021-eular.577

\section{POS0023 \\ RACIAL/ETHNIC AND REGIONAL DIFFERENCES IN MULTIMORBIDITY BETWEEN PATIENTS WITH RHEUMATOID ARTHRITIS AND COMPARATORS IN A LARGE NATIONWIDE US STUDY}

H. J. Dykhoff ${ }^{1}$, E. Myasoedova ${ }^{2,3}$, M. Peterson ${ }^{2}$, J. M. Davis $\mathrm{II}^{2}$, V. Kronzer ${ }^{2}$, C. Coffey ${ }^{2}$, T. Gunderson ${ }^{3}$, C. S. Crowson ${ }^{2,3} .{ }^{1}$ Mayo Clinic, Robert D. and Patricia E. Kern Center for the Science of Health Care Delivery, Rochester, 
United States of America; ${ }^{2}$ Mayo Clinic, Division of Rheumatology, Rochester, United States of America; ${ }^{3}$ Mayo Clinic, Department of Quantitative Health Sciences, Rochester, United States of America

Background: Patients with rheumatoid arthritis (RA) have an increased burden of multimorbidity. Racial/ethnic disparities have also been associated with an increased burden of multimorbidity.

Objectives: We aimed to compare multimorbidity among different racial/ethnic groups and geographic regions of the US in patients with RA and comparators without RA.

Methods: We used a large longitudinal, real-world data warehouse with de-identified administrative claims for commercial and Medicare Advantage enrollees, to identify cases of RA and matched controls. Cases were defined as patients aged $\geq 18$ years with $\geq 2$ diagnoses of RA in January 1, 2010 - June 30, 2019 and $\geq 1$ prescription fill for methotrexate in the year after the first RA diagnosis. Controls were persons without RA matched 1:1 to RA cases on age, sex, census region, calendar year of index date (corresponding to the date of second diagnosis code for RA), and length of prior medical/pharmacy coverage. Race was classified as non-Hispanic White (White), non-Hispanic Black (Black), Asian, Hispanic, or other/unknown, based on self-report or derived rule sets. Multimorbidity (2 or more comorbidities) was defined using 25 chronic comorbidities from a combination of the Charlson and Elixhauser Comorbidity Indices assessed during the year prior to index date. Rheumatic comorbidities were not included. Logistic regression models were used to estimate odds ratios (OR) with $95 \%$ confidence intervals $(\mathrm{Cl})$.

Results: The study included 16,363 cases with RA and 16,363 matched non-RA comparators (mean age 58.2 years, $70.7 \%$ female for both cohorts). Geographic regions were the same in both cohorts: $50 \%$ South, $26 \%$ Midwest, $13 \%$ West, and $11 \%$ Northeast. Race/ethnicity was not part of the matching criteria and varied slightly between the cohorts: among RA (non-RA) patients, $74 \%$ (74\%) were White, $11 \%$ (9\%) Hispanic, 10\% (9\%) Black, 3\% (4\%) Asian, and 3\% (4\%) other/unknown. Patients with RA had more multimorbidity than non-RA subjects $(51.3 \%$ vs $44.8 \%)$. Multimorbidity comparisons across US geographic regions were similar in both cohorts, with comparable multimorbidity levels for patients in the West and Midwest and higher levels for those in the Northeast and South (Figure 1). Among the non-RA patients, $43.5 \%$ of Whites experienced multimorbidity, compared to $33.9 \%$ of Asians, $46.1 \%$ of Hispanics, and $58.4 \%$ of Blacks. These associations remained after adjustment for age, sex, and geographic region, with significantly lower multimorbidity among Asians (OR: 0.81; 95\% Cl: 0.67-0.99) and significantly higher multimorbidity among Hispanics (OR: $1.21 ; 95 \% \mathrm{Cl}: 1.07-1.37$ ) and Blacks (OR: $1.74 ; 95 \% \mathrm{Cl}$ : $1.54-1.97$ ), compared to Whites in the non-RA cohort. Among the RA patients, racial/ethnic differences were less pronounced; $50.6 \%$ of Whites, $42.8 \%$ of Asians, $48.8 \%$ of Hispanics, and $58.4 \%$ of Blacks experienced multimorbidity. Adjusted analyses revealed no significant differences in multimorbidity for Asians (OR: $0.88 ; 95 \% \mathrm{Cl}: 0.70-1.08$ ) and Hispanics (OR: $1.06 ; 95 \% \mathrm{Cl}: 0.95-1.19)$ and a less pronounced increase in multimorbidity among Blacks (OR: 1.32; $95 \% \mathrm{Cl}$ : 1.17-1.49) compared to Whites in the RA cohort.

Conclusion: This large nationwide study showed increased occurrence of multimorbidity in RA versus non-RA patients and in both cohorts for residents of the Northeast and South regions of the US. Racial/ethnic disparities in multimorbidity were more pronounced among patients without RA compared to RA patients. This indicates the effects of RA and race/ethnicity on multimorbidity do not aggregate. The underlying mechanisms for these associations require further investigation.

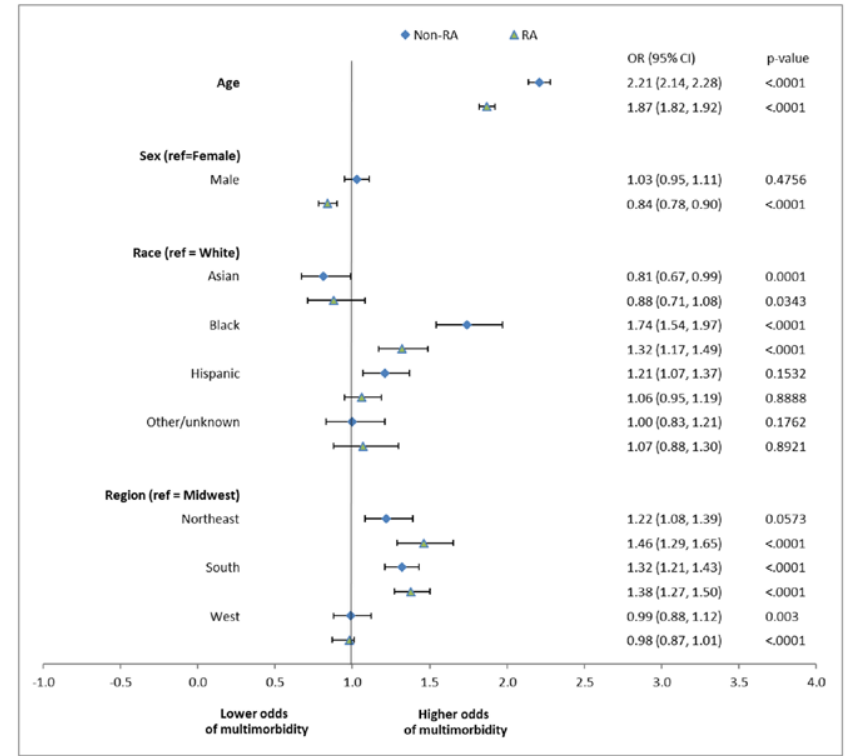

Figure 1. Logistic regression models comparing multimorbidity levels in RA and non-RA cohorts.

Disclosure of Interests: Hayley J. Dykhoff: None declared, Elena Myasoedova: None declared, Madeline Peterson: None declared, John M Davis III Grant/ research support from: Research grant from Pfizer, Vanessa Kronzer: None declared, Caitrin Coffey: None declared, Tina Gunderson: None declared, Cynthia S. Crowson: None declared.

DOI: 10.1136/annrheumdis-2021-eular.1610

\section{POS0024}

ESTIMATED PREVALENCE, INCIDENCE AND HEALTHCARE COSTS OF SJÖGREN'S SYNDROME IN FRANCE: A NATIONAL CLAIMS-BASED STUDY

R. Seror ${ }^{1}$, L. Chiche ${ }^{2}$, G. Desjeux ${ }^{3}$, J. Zhuo ${ }^{4}$, B. Bregman ${ }^{5}$, V. VannierMoreau ${ }^{6}$, V. Devauchelle-Pensec ${ }^{7} .{ }^{1}$ Université Paris-Sud, AP-HP, Hôpitaux Universitaires Paris-Sud, N/A, Paris, France; ${ }^{2}$ Hôpital Européen Marseille, Unité de medicine interne et recherche clinique, Marseille, France; ${ }^{3}$ Sanoia, eHealth services, Aubagne, France; ${ }^{4}$ Bristol Myers Squibb, Worldwide Health Economics and Outcomes Research, Princeton, United States of America; ${ }^{5}$ Bristol Myers Squibb, Health Economics and Public Health, RueilMalmaison, France; ${ }^{6}$ Bristol Myers Squibb, Medical Affairs, France, RueilMalmaison, France; ${ }^{7} \mathrm{CHU}$ de Cavale Blanche, Rheumatology Department, Brest, France

Background: Sjögren's syndrome (SS) is a chronic, systemic autoimmune disorder characterised by oral and ocular dryness related to lymphocytic infiltration of exocrine glands. ${ }^{1-3}$ Extra-glandular manifestations may include fatigue, musculoskeletal pain and glomerulonephritis. ${ }^{1,3}$ SS can present as primary SS (pSS) 